\title{
Competencias en geometría en estudiantes de educación básica primaria
}

\author{
Competences in geometry in students of primary basic education
}

\author{
- JUAN CARLOS MARTÍNEZ HUERTAS \\ jucama84@gmail.com \\ Universidad Pedagógica y Tecnológica de Colombia
}

Artículo recibido en septiembre 2018 / Arbitrado en octubre 2018 / Publicado en enero 2019

Resumen Esta investigación tuvo como objetivo diagnosticar las competencias en geometría, de los estudiantes de las escuelas públicas del Municipio Ramiriquí, Departamento de Boyacá, Colombia, para 2019. Fue una investigación descriptiva, con diseño de campo transeccional contemporáneo multivariable. El abordaje fue cosmológico, exógeno y ético. La población estuvo conformada por 261 estudiantes. Se realizó un muestreo probabilístico estratificado según las instituciones y las sedes. Para la recolección de datos se diseñó una prueba de competencias en geometría para ser aplicada a los estudiantes. En el análisis de resultados descriptivos de cada evento se utilizaron porcentajes, mínimo, máximo y mediana, con gráficos de caja y bigotes. Los resultados permitieron evidenciar una tendencia en cuanto a la dificultad con establecer estrategias para la solución de problemas relativos a la medida del perímetro de figuras planas. También se encontró que los estudiantes prefieren realizar ejercicios prácticos que sean aplicables en algún contexto específico a realizar tareas netamente teóricas.

Palabras clave: Educación; educación básica; competencias; competencias en geometría

\begin{abstract}
This research aimed to diagnose the geometry competences of the students of the public schools of the Ramiriquí Municipality, Department of Boyacá, Colombia, for 2019. It was a descriptive investigation, with a multivariable contemporary transectional field design, and the approach was cosmological, exogenous and ethical. The population was made up of 261 students. A stratified probability sampling was carried out according to the institutions and the headquarters. For data collection, a geometry skills test was designed to be applied to students. In the analysis of descriptive results of each event, percentages, minimum, maximum and median were used, with box plots and whiskers. The results showed a trend regarding the difficulty with establishing strategies for solving problems related to the measurement of the perimeter of flat figures. It was also found that students prefer to carry out practical exercises that are applicable in some specific context to carrying out purely theoretical tasks.
\end{abstract}

Keywords: Education; elementary education; competences; competences on geometry 
INTRODUCCIÓN

La educación sigue siendo uno de los grandes aportes de la humanidad a través de los tiempos, es por esto que la academia, las instituciones, las sociedades y los gobiernos buscan cada día el mejoramiento, la calidad, la cobertura y todos los componentes que fortalezcan el conocimiento para la búsqueda del mejoramiento de la calidad de vida de los seres humanos.

En cuanto a la educación básica primaria, Fabbricatore (2009) destaca que esta etapa de la Educación Básica es un período donde los ejes transversales y las disciplinas del saber aparecen totalmente integrados, por esta razón se le reconoce como una etapa de integración, en la cual el aprendizaje se da de una manera globalizada.

La educación basada en competencias exige la búsqueda constante de estrategias que faciliten el aprendizaje de los estudiantes, por consiguiente indagar y aplicar con acierto nuevas perspectivas didácticas, encaminadas hacia el desarrollo integral del estudiante debe ser tarea diaria de quienes se ocupan de planear, facilitar, motivar y hacer seguimiento a las tareas del aula de clase.

Es necesario hacer un seguimiento a los aprendizajes de los estudiantes de forma continua, teniendo en cuenta la diversidad de los estudiantes existente en la cotidianidad del aula de clase, en procura de mejorar la calidad educativa Colombiana para contribuir a que el país se convierta en el más educado en América Latina a 2025.

En esta investigación se presenta un diagnóstico de la situación actual del aprendizaje de los estudiantes en cuanto a las competencias en geometría.

Según Marchesi, Tedesco y Coll (2015) el mundo experimenta cambios en todos los órdenes de la vida social, cultural, económica y política. El fenómeno de la globalización, caracterizado por el libre comercio, el libre tránsito de capitales y de información, exige que los sistemas educativos aseguren la calidad de la enseñanza y de los aprendizajes. En el proceso de globalización se fortalece una nueva sociedad cuyo valor principal se encuentra en el conocimiento, tanto por su influencia en los procesos de producción, como en otros ámbitos de la vida humana.

Las matemáticas forman parte del currículo de todos los niveles de educación, por consiguiente es indispensable su estudio, y se tienen en cuenta diferentes miradas que existieron, existen y puedan existir en cuanto su enseñanza, pues en ocasiones aprender o enseñar matemáticas puede resultar tarea tediosa, más cuando los estudiantes se han llenado de barreras producto de la herencia cultural que son transmitidas de una generación a otra en cuanto a que se perciben las matemáticas como muy difíciles, claro está que cada persona habla desde su experiencia con esta disciplina, que en última instancia es útil en todos los campos del conocimiento.

Una de las principales ramas de la matemática es la geometría. Según Godino y Ruiz (2002), esta disciplina se ocupa de una clase especial de objetos que se designan con palabras como, punto, recta, plano, triángulo, polígono, poliedro, etc. Tales términos y expresiones hacen referencia a 
figuras geométricas, las cuales son consideradas como abstracciones, conceptos, entidades ideales o representaciones generales de una categoría de objetos.

Prosiguiendo con lo planteado por Godino y Ruiz (2002), mediante la geometría el ser humano refleja en su quehacer diario y en sus obras de arte imágenes ideales que obtiene producto de la observación de la naturaleza; realiza objetos de cerámica, dibujos, edificios y los más diversos utensilios, y proyecta en ellos las figuras geométricas que ha perfeccionado en la mente.

En este sentido se puede notar que la geometría se aplica en la cotidianidad desde diversas áreas del conocimiento, especialmente en cuanto a las artes, sin dejar a un lado las disciplinas relacionadas con la construcción, que es quizás donde más se aplica, aunque muchas veces quienes la utilizan no valoran su importancia en el trabajo que realizan.

En la actualidad se genera continuamente una gran cantidad de información relacionada con la geometría, la cual se presenta de diversas formas: gráfica, numérica y verbal. Por esta razón, es importante que desde la educación básica primaria se desarrolle el pensamiento lógico matemático en los estudiantes, basado en la construcción de un conjunto de competencias que puedan ser utilizadas en cualquier situación que se les presente, ya sea escolar o no.

León y Barcia (2016) manifiestan que la etapa que abarca la educación primaria tiene gran importancia en el proceso de enseñanza-aprendizaje de la geometría, pues es en ella cuando se comienza a desarrollar en los escolares la capacidad de interiorizar las propiedades geométricas observadas y la formación de su vocabulario geométrico.

El MEN de Colombia (2004) señala que de acuerdo con los estándares de competencias esperados, en los resultados de las pruebas SABER, los estudiantes evidencian un mayor desarrollo de las competencias en lenguaje, en comparación con las competencias matemáticas, tanto en colegios públicos como privados.

Según Castellanos (2001), Colombia participó en PISA (Programme for International Student Assessment) por primera vez en 2006, siendo los resultados en Matemáticas los más preocupantes. La puntuación promedio fue sólo 370 puntos. Más del $70 \%$ de los estudiantes se sitúan en los niveles 1 y 2 y ninguno en los dos niveles superiores. La puntuación promedio estuvo muy por debajo del promedio general y por debajo de la media entre los países de América Latina y el Caribe.

En cuanto a las pruebas TIMSS (Trends in International Mathematics and Science Study), Castellanos (2001) expresa que Colombia participó por primera vez durante el año 1995, obtuvo uno de los últimos lugares en el ranking internacional. La siguiente participación ocurrió en el año 2007, donde el promedio global de los estudiantes colombianos de cuarto grado fue 355 puntos. Una situación similar se observa en octavo, en donde el promedio global de Colombia fue 380. En ambos grados nuestro promedio fue significativamente inferior al promedio TIMSS (500 puntos de media internacional). 
En cuarto grado, el $69 \%$ de los niños mostró logros inferiores; el 22\% se ubicó en el nivel bajo; tan solo un $7 \%$ en el medio, $2 \%$ en el alto y ninguno en el avanzado. En octavo el $61 \%$ tuvo logros inferiores, el $28 \%$ en el nivel bajo, en tanto que el $9 \%$ en el medio, el $2 \%$ en el alto y ninguno en el avanzado.

A partir de cifras reveladas por la Secretaria de Educación del departamento de Boyacá (SEDBOYACA, 2018), en el área de matemáticas, el mayor porcentaje de estudiantes se ubican en un nivel mínimo según los reportes históricos de las pruebas SABER. En el nivel mínimo en el año 2012 fue del 33\%, en el año 2013 disminuyo al 31\%, en el año 2014 disminuyo al 29\%, en el año 2015 aumento al 31\%, en el año 2016 se mantuvo en el $31 \%$, y permaneció estable, en el año 2017 obtuvo un 32\% desmejorando en $1 \%$.

Se encontró que las principales dificultades de la geometría en educación básica primaria están asociadas fundamentalmente al reconocimiento de figuras y cuerpos a partir de sus propiedades, a la habilidad para establecer relaciones entre las figuras, y a la solución de problemas geométricos, fundamentalmente cuando se requiere de aplicar o encontrar una vía novedosa para la solución de la situación planteada (León, 2008).

Según Gómez y Perry (1996), en Colombia existe una problemática alrededor de la calidad de la formación matemática de los estudiantes en los colegios. Tal problemática suele asociarse de manera exclusiva con algunos indicadores como la alta mortalidad académica y la deserción escolar.
Con base en la problemática planteada, es importante plantear la siguiente pregunta de investigación: ¿Cuáles son las competencias en geometría, de los estudiantes de las escuelas públicas del Municipio Ramiriquí, Departamento de Boyacá, Colombia, para 2019?

Dicha interrogante conllevó a la valoración de un conjunto de posturas teóricas como las que expresan que son varios los avances y esfuerzos los que han realizado los gobiernos, y la misma sociedad, para mejorar los sistemas educativos, con la creación de formatos, lineamientos institucionales, estándares internacionales, formación docente y muchos otros aspectos destinados a promover la calidad educativa, pero aún hay un camino largo para lograr una educación que, además de tener cobertura, sea de calidad. En el caso de Colombia los distintos gobiernos han intentado mejorar la calidad en la educación buscando la formación de personas capaces de desarrollar una serie de competencias que ayuden a desenvolverse y mantener una mejora continua de la de la sociedad.

La matemática es una disciplina que tiene aplicación en todos los campos del conocimiento; la matemática es una herramienta muy importante que ayuda al desarrollo de las demás áreas del conocimiento, Buró (2002) afirma que los diferentes tipos de conocimiento se seleccionan dentro de una estructura de áreas que incluyen la lengua materna y las matemáticas como herramientas básicas para el aprendizaje y también las demás áreas del conocimiento y de la formación humana. 
Esta área del saber constituye el armazón sobre el que se construyen los modelos científicos, toman parte en el proceso de modelización de la realidad, y en muchas ocasiones han servido como medio de validación de estos modelos. Por ejemplo, han sido cálculos matemáticos los que permitieron, mucho antes de que pudiesen ser observados, el descubrimiento de la existencia de los últimos planetas de nuestro sistema solar (Godino y Ruiz, 2002).

La geometría es una de las ramas de la matemática y se ocupa del estudio de la extensión, la forma de medirla, las relaciones entre puntos, líneas, ángulos, planos y figuras, y la manera cómo se miden. Para Godino y Ruiz (2002), la geometría se ocupa de una clase especial de objetos que designados con palabras como, punto, recta, plano, triángulo, polígono, poliedro, etc. Tales términos y expresiones designan "figuras geométricas", las cuales son consideradas como abstracciones, conceptos, entidades ideales o representaciones generales de una categoría de objetos.

Muchas profesiones, además de los matemáticos, arquitectos e ingenieros necesitan y usan la Geometría: albañiles, ceramistas, artesanos (objetos de taracea, trabajos de cuero, repujados de latón, tejedores de alfombras, bordadoras, encajes de bolillos, etc.) decoradores, coreógrafos, diseñadores de muebles, etc. Todos ellos de una forma más o menos consciente, utilizan el espacio y las formas geométricas, (Godino y Ruiz, 2002, p. 457).

Saber geometría es más que reconocer figuras y cuerpos por sus nombres: es resolver problemas geométricos apoyándose en propiedades conocidas de figuras y cuerpos; en situaciones que, generalmente, son intramatemáticas, geométricas y que cuentan o no con apoyo gráfico. Su solución es lo que da sentido a la enseñanza de la Geometría (León y Barcia, 2016).

Para Godino y Ruiz (2002), la geometría se ocupa de una clase especial de objetos que designados con palabras como, punto, recta, plano, triángulo, polígono, poliedro, etc. Tales términos y expresiones designan "figuras geométricas", las cuales son consideradas como abstracciones, conceptos, entidades ideales o representaciones generales de una categoría de objetos.

Por otra parte, D’Amore (2005) señala que el aprendizaje de las matemáticas que no se basa solo en la construcción del conocimiento sino que consta de cuatro tipologías distintas, aprendizaje conceptual, aprendizaje de estrategias (resolver, demostrar...) aprendizaje algorítmico (calcular, operar...) y el aprendizaje comunicativo.

La educación basada en competencias es una nueva orientación educativa que pretende dar respuestas a la sociedad, según Tobón (2006), las competencias buscan reforzar y contribuir a que las personas sean emprendedoras, primero como seres humanos y en la sociedad, y después en lo laboral-empresarial para mejorar y transformar la realidad

Para Godino y Ruiz (2002), la palabra competencia se refiere a un saber hacer específico. Generalmente tener competencia es equivalente a tener conocimiento práctico sobre algo; se usa habitualmente referido a destrezas manipulativas o procedimentales. 
EI MEN (2006) considera que las competencias son un conjunto de conocimientos, habilidades, actitudes, comprensiones y disposiciones cognitivas, socio afectivas y psicomotoras apropiadamente relacionadas entre sí para facilitar el desempeño flexible, eficaz y con sentido de una actividad en contextos relativamente nuevos y retadores.

En relación a las competencias, Tobón (2006) distingue tres tipos de competencias fundamentales:

- Competencias cognitivas, definidas como la puesta en acción-actuación de un conjunto de herramientas necesarias para procesar la información de manera significativa acorde con las expectativas individuales, las propias capacidades y los requerimientos de una situación en particular

\section{- Competencias procedimentales,} definidas como el saber hacer consiste en saber actuar con respecto a la realización de una actividad o la resolución de un problema, comprendiendo el contexto y teniendo como base la planeación.

- Competencias actitudinales, definidas como disposiciones afectivas a la acción. Constituyen el motor que impulsa al comportamiento en los seres humanos. Inducen a la toma de decisiones y a desplegar un determinado tipo de comportamiento acorde con las circunstancias del momento. No son observables de forma directa. Se detectan a partir de cómo se comportan las personas, qué dicen y cómo es su comunicación no verbal (gestos, posiciones corporales, señalizaciones, etc.)
A pesar de que el modelo por competencias ha aportado grandes avances en el campo económico y productivo y ha intentado desarrollar un modelo más eficiente este modelo esta desligado de cada uno de los contextos, de las necesidades, pues no se trata solamente del desarrollo de la lectoescritura y de una serie de operaciones matemáticas que no involucran la realidad, pues en ocasiones se debe cumplir unos estándares internacionales que muy seguramente no son aplicables a la realidad.

Según Godino y Ruiz (2002), las competencias que deben manejar los niños al finalizar la educación básica primaria, están relacionadas con formas geométricas y situación en el espacio, procedimientos y actitudes. Algunas de estas competencias son:

- La situación en el espacio (distancias, ángulos y giros, y sistema de coordenadas cartesianas)

- Relación entre elementos geométricos tales como paralelismo y perpendicularidad.

- La representación elemental del espacio a través de planos, mapas, y maquetas.

- Relaciones entre formas planas y espaciales

- Transformaciones en el plano teniendo en cuenta regularidades y simetrías.

- Descripción de la posición de un objeto en el espacio según puntos de referencia.

- Interpretación y descripción de croquis, planos, maquetas y mapas.

- Comparación y clasificación de figuras y cuerpos geométricos según propiedades y criterios. 
- Formación de figuras planas y cuerpos geométricos a partir composición y descomposición.

- Búsqueda de elementos de regularidad y simetría en figuras y cuerpos geométricos.

- Utilización de sistemas de referencia y de la representación espacial en actividades cotidianas.

- Gusto por la elaboración y presentación cuidadosa de construcciones de objetos geométricos.

- Precisión y cuidado en el uso de instrumentos de dibujo.

- Interés y perseverancia en la búsqueda de soluciones a problemas relacionados con la utilización del espacio.

Las consecuencias de no superar a tiempo dificultades respecto a las competencias geométricas en la educación básica primaria, ocasionan que en los siguientes niveles de la educación secundaria los estudiantes tengan problemas para asimilar temáticas relacionadas con representación objetos tridimensionales desde diferentes posiciones y vistas, identificar y describir figuras relacionadas con cuerpos generados por cortes rectos y transversales de objetos tridimensionales, clasificar polígonos en relación con sus propiedades, predecir y comprar los resultados de aplicar transformaciones rígidas (traslaciones, rotaciones, reflexiones) y homotecias (ampliaciones y reducciones) sobre figuras bidimensionales en situaciones matemáticas y en el arte, resolver y formular problemas que involucren relaciones $y$ propiedades de semejanza y congruencia usando representaciones visuales, resolver y formular problemas usando modelos geométricos e identificar características de localización de objetos en sistemas de representación cartesiana y geográfica (MEN, 2006).

\section{MÉTODO}

Este estudio fue una investigación descriptiva. Se realizó mediante un diseño de campo. En cuanto a la temporalidad, el diseño fue transeccional contemporáneo. Según el número de eventos, el diseño fue univariable. Se realizó en el municipio de Ramiriquí municipio colombiano, capital de la Provincia de Márquez, ubicado en el departamento de Boyacá a 30 kilómetros de Tunja, la capital del departamento. Este municipio está constituido en su zona rural por 24 veredas, y en su zona urbana por 8 barrios. La población de estudio estuvo conformada por los estudiantes, 261 de los grados cuarto y quinto de Básica primaria, pertenecientes a las tres instituciones oficiales del municipio de Ramiriquí en el año 2019, como también los docentes que orientan la asignatura de matemáticas en dichos grados.

Con respecto a la técnica de muestreo se realizó uno estratificado, para garantizar que todas las instituciones tuviesen representación en la muestra siguiente: 
Tabla 1. Descripción de la muestra de estudio

\begin{tabular}{|c|c|c|c|c|c|c|c|c|c|}
\hline I.E & Sede & Cuarto & Quinto & Total & Total & $\%$ & Cuarto & Quinto & Estrato \\
\hline \multirow{4}{*}{ El Escobal } & Escobal & 20 & 23 & 43 & \multirow[b]{3}{*}{58} & \multirow[b]{3}{*}{$22,22 \%$} & 8 & 8 & 16 \\
\hline & Hortigal & 1 & 3 & 4 & & & 1 & 1 & 2 \\
\hline & $\begin{array}{l}\text { San } \\
\text { Antonio }\end{array}$ & 1 & 0 & 1 & & & 1 & 0 & 1 \\
\hline & Guayabal & 3 & 7 & 10 & \multirow{5}{*}{27} & \multirow{4}{*}{$10,34 \%$} & 1 & 2 & 3 \\
\hline \multirow{2}{*}{ Naguata } & Naguata & 3 & 9 & 12 & & & 1 & 3 & 4 \\
\hline & Hervideros & 2 & 3 & 5 & & & 1 & 1 & 2 \\
\hline \multirow{5}{*}{$\begin{array}{l}\text { José } \\
\text { Ignacio } \\
\text { de } \\
\text { Marquez } \\
\text { Total }\end{array}$} & Fernández & 4 & 2 & 6 & & & 1 & 1 & 2 \\
\hline & Santuario & 2 & 2 & 4 & & \multirow{3}{*}{$67,43 \%$} & 1 & 1 & 2 \\
\hline & $\begin{array}{l}\text { Antonio } \\
\text { Ricaute }\end{array}$ & 69 & 96 & 165 & \multirow{2}{*}{176} & & 26 & 36 & 62 \\
\hline & Farquenta & 8 & 3 & 11 & & & 3 & 1 & 4 \\
\hline & & 113 & 148 & 261 & 261 & & 44 & 54 & 98 \\
\hline
\end{tabular}

Para diagnosticar las competencias en geometría se utilizó como técnica la encuesta y como instrumento una prueba de competencias en geometría con 50 ítems de respuesta cerrada. La prueba abarcó competencias cognitivas, actitudinales y procedimentales.

Para las competencias cognitivas se incluyeron 11 ítems, que se puntuaron como 1 y 0 según fuesen correctas $o$ incorrectas las repuestas, respectivamente, el puntaje bruto fue de 11 puntos. El bloque de las competencias procedimentales tenía 23 ítems, donde cada ítem puntuaba 0 ó 1, según.

La dimensión relacionada con las competencias actitudinales constó de 16 ítems con 3 alternativas de respuesta: nunca, algunas veces y siempre, donde cada ítem puntuaba de 0,1 ó 2 . El puntaje más alto se asignó a las respuestas que indicaban actitud más favorable. En esta dimensión el puntaje bruto fue de 32 puntos.

La escala total de la prueba competencias en geometría dio un puntaje máximo total de 66 puntos. Para el total y para las dimensiones se calculó el puntaje transformado en una escala de 100 puntos. El índice de validez de 0,70 y la confiabilidad fue de 0,77

Se utilizó, como técnica de análisis de datos, el análisis descriptivo por medio de métodos estadísticos con mediana, frecuencias y porcentajes. Para interpretar los puntajes obtenidos se utilizaron las categorías que se muestran en la Tabla 2.

Tabla 2. Tabla de interpretación de puntajes

\begin{tabular}{ll}
\hline Intervalo & Categoría \\
\hline De 0 hasta 19,99 & Insuficiente \\
De 20 hasta 39,99 & Bajo \\
De 40 hasta 59,99 & Aceptable \\
De 60 hasta 79,99 & Satisfactorio \\
De 80 hasta 100 & Avanzado \\
\hline
\end{tabular}




\section{RESULTADOS}

El grupo de estudiantes obtuvo una mediana de 52,79 puntos sobre 100 puntos, que al ser comparada con la Tabla de intervalos y categorías de interpretación, se ubicó en la categoría aceptable. Esto significa que, en términos generales, presentaron un nivel mínimo aceptable en cuanto al desarrollo de competencias en geometría.

Tabla 3. Media obtenida por el grupo de estudiantes en competencias en geometría

$\begin{array}{lll}N & \text { Válidos } & 98 \\ \text { Perdidos } & 0 \\ \text { Mediana } & 52,27 \\ \text { Máximo } & 78,79 \\ \text { Percentiles } & \text { Mínimo } & 19,70 \\ & 25 & 43,5600 \\ & 50 & 52,2750 \\ 75 & 62,1200\end{array}$

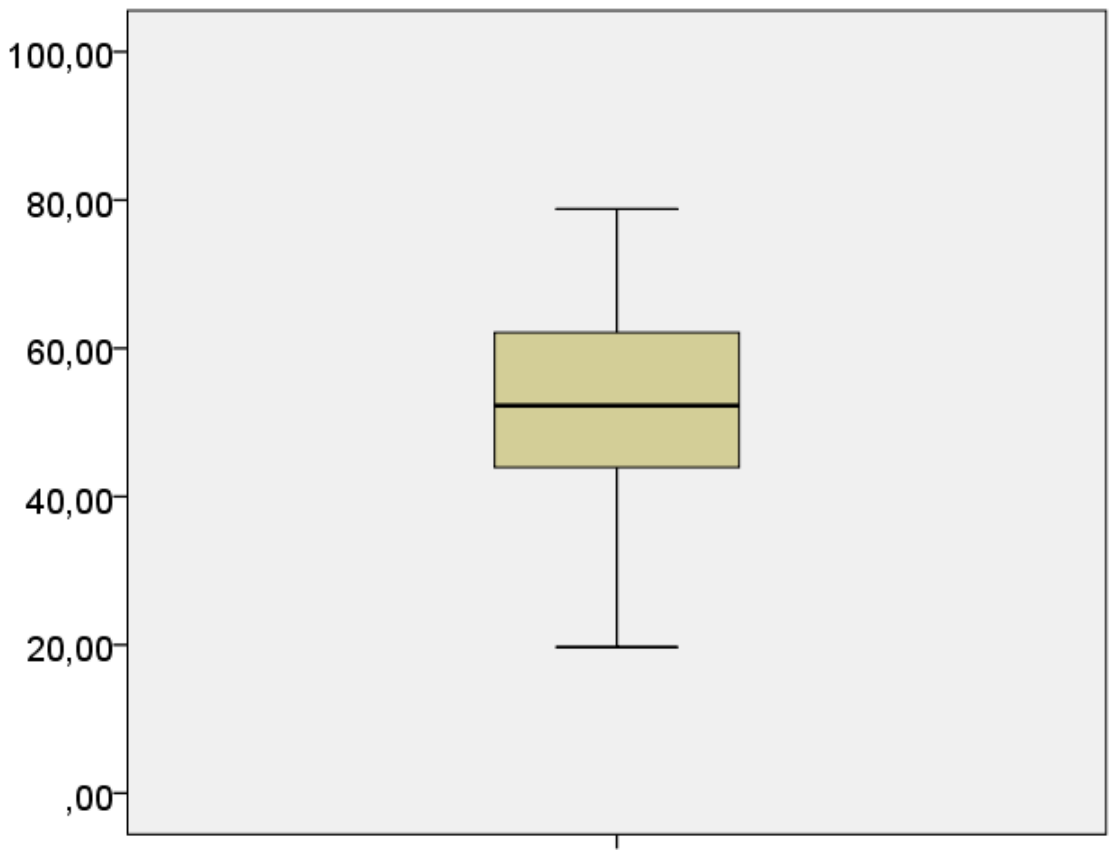

Gráfico 1. Mediana de las competencias en geometría

El puntaje mínimo fue de 19,70 puntos, ubicado en la categoría insuficiente y el puntaje máximo fue de 78,79 puntos, ubicado en la categoría satisfactorio. No se encontraron casos en la categoría avanzado. Tampoco se encontraron casos atípicos. Esto significa que los estudiantes están alcanzando el mínimo de logro requerido para aprobar, pero no dominan gran parte de las competencias

Las competencias en geometría están conformadas por tres dimensiones: cognitiva, procedimental y actitudinal. En el gráfico 2 se aprecian las medianas de las diferentes dimensiones del evento competencias en geometría. El puntaje 
obtenido en la dimensión competencias cognitivas fue de 45,45; para la dimensión competencias procedimentales fue de 52,17; y en la dimensión competencias actitudinales fue de 56,25 , en una escala de 100 puntos. Las competencias actitudinales obtuvieron la mediana más alta, aunque la diferencia no es grande. En segundo lugar, se ubicó la dimensión competencias procedimentales y por último se ubicó la dimensión competencias cognitivas, lo cual muestra que los estudiantes tienen más facilidad para realizar ejercicios prácticos que se puedan aplicar en alguna situación específica, que para asimilar contenidos abstractos. El grupo resultó ser heterogéneo en las tres dimensiones.

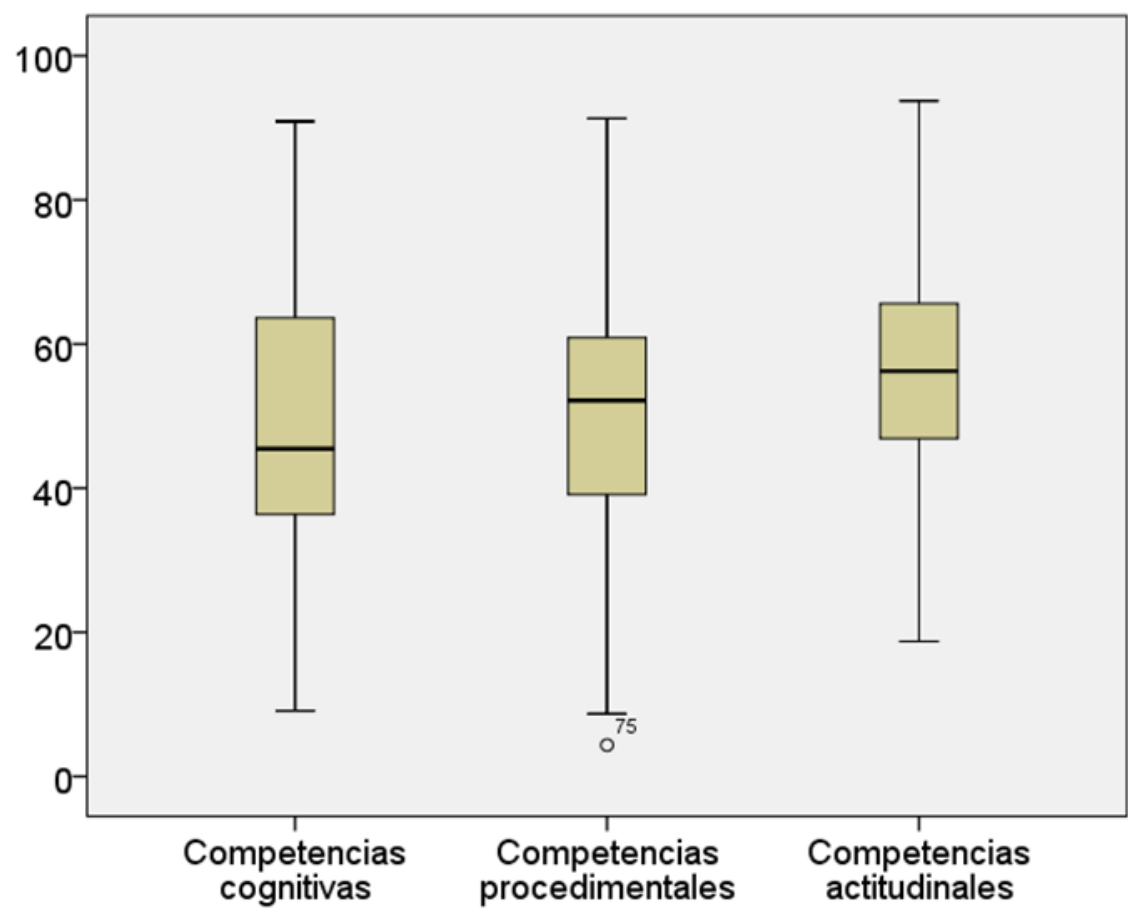

Gráfico 2. Mediana competencias en geometría por dimensiones

Las competencias cognitivas que resultaron ser más favorables para los estudiantes, teniendo en cuenta el porcentaje de respuestas correctas o incorrectas por ítem, fueron las siguientes:

- Comparan diferentes figuras a partir de las medidas de sus lados, describen el comportamiento que del área cuando se modifica alguno de sus lados, realizan comparaciones entre una figura tridimensional y el desarrollo de alguna de sus caras.

- Muestran habilidad para resolver situaciones que tienen que ver con el análisis de las propiedades de las figuras geométricas.

- En cuanto a lo menos favorable, tienen dificultad para describir el comportamiento que del perímetro cuando se modifica alguno de sus lados, para describir figuras bidimensionales y 
tridimensionales, y para reconocer posibles relaciones entre las magnitudes del área y el perímetro de una figura.

Las competencias procedimentales que resultaron ser más favorables para los estudiantes, teniendo en cuenta los porcentajes de respuestas correctas o incorrectas por ítem, fueron las siguientes:

- Clasifican polígonos según sus propiedades.

- Clasifican objetos según su posición relativa.

- Utilizan figuras bidimensionales y tridimensionales para representar situaciones reales.

- Representan en el plano cartesiano la posición con direcciones cardinales.

En general se evidenciaron fortalezas para la descripción de diferentes figuras geométricas y para representar situaciones reales que involucran los puntos cardinales como coordenadas.

En cuanto a las competencias procedimentales menos favorables para los estudiantes, según los porcentajes de respuestas correctas o incorrectas por ítem, los resultados son los siguientes:

- No establecen estrategias para la solución de problemas relativos a la medida de perímetro de figuras planas.

- No relacionan coordenadas de origen con las coordenadas finales de un objeto que ha efectuado un movimiento.

- No resuelven problemas de trasformaciones en el plano.

- No utilizan el sistema de coordenadas para ubicar figuras planas.
Se evidencia una dificultad para establecer estrategias para la solución de problemas relativos a la medida del perímetro de figuras planas.

En lo que respecta a las competencias actitudinales, el 13,2\% del grupo tiene actitudes entre negativas y muy negativas, y sólo el $7,1 \%$ tiene inclinaciones favorables hacia la geometría. Se percibió el agrado de los estudiantes por el trabajo con figuras geométricas en el plano, guardando propiedades que las caracterizan. También se apreció que a los estudiantes les general les resulta divertido encontrar referencias en el plano cartesiano.

En cuanto a las competencias actitudinales que resultaron ser menos favorables para los estudiantes, tiene que ver con el ser cuidadoso en el uso de unidades de medida y en las construcciones geométricas, así como en la falta de disposición a usar la imaginación para visualizar cómo sería una posible representación del objeto geométrico.

Con respecto a las competencias en geometría, la mayor parte de los estudiantes se ubicaron dentro de los criterios mínimos de aprobación, lo que significa que tenían un manejo muy precario en cuanto a la capacidad para integrar los conocimientos, habilidades, destrezas, prácticas y acciones relacionadas con el dominio de la geometría. Esto no cumple con lo deseado, pues el MEN (2006), afirma que desarrollar satisfactoriamente las competencias implica integrar conocimientos, potencialidades, habilidades, destrezas, prácticas y acciones, manifestadas a través de los desempeños o acciones de aprendizaje propuestas en cada área. 
El no desarrollar competencias geométricas en la educación básica primaria puede dificultar la asimilación de competencias en los siguientes niveles de escolaridad relacionados con tareas como representar objetos tridimensionales desde diferentes posiciones y vistas, identificar y describir figuras y cuerpos generados por cortes rectos y transversales de objetos tridimensionales, clasificar polígonos en relación con sus propiedades, predecir y comprar los resultados de aplicar transformaciones rígidas (traslaciones, rotaciones, reflexiones) y homotecias (ampliaciones y reducciones) sobre figuras bidimensionales en situaciones matemáticas $y$ en el arte, resolver y formular problemas que involucren relaciones y propiedades de semejanza y congruencia usando representaciones visuales, resolver $y$ formular problemas usando modelos geométricos e identificar características de localización de objetos en sistemas de representación cartesiana y geográfica (MEN, 2006).

Otras implicaciones que traen las debilidades de las competencias en geometría, en la educación primaria, tienen que ver con la dificultad para acceder carreras profesionales relacionadas con el razonamiento espacial como lo son la arquitectura, las artes e ingenierías entre otras.

En cuanto a dimensión competencias cognitivas los resultados arrojaron que un $73,5 \%$ de los estudiantes, del grupo de estudiantes presentan dificultades en cuanto a las competencias cognitivas en geometría, dentro de las cuales se recalcan las relacionadas con la interpretación de la variación del área y el perímetro de figuras planas, pues se logró evidenciar que los estudiantes tienen la tendencia a crear dependencia entre las dos magnitudes en cuanto a que una afecta el comportamiento de la otra. Lori, Fandiño y D'Amore (2013) han realizado vastas investigaciones sobre esta temática y encontraron resultados similares.

Dentro de las competencias cognitivas que tuvieron mayor acierto están las relacionadas con la descripción de diferentes figuras geométricas y la representación de situaciones que involucran puntos cardinales. En cuanto a las competencias procedimentales que resultaron ser menos favorables se evidencia una tendencia en cuanto a la dificultad para establecer estrategias para la solución de problemas relativos a la medida del perímetro de figuras planas.

En la dimensión competencias actitudinales se evidenció un nivel medio de agrado de los estudiantes por el trabajo con figuras geométricas en el plano, guardando propiedades que las caracterizan, mientras que las competencias actitudinales que resultaron ser menos favorables para los estudiantes, tienen que ver con el proceso de visualización de figuras geométricas, ya que tienen poca disposición para imaginar representaciones del proceso geométrico y para trabajar de manera cuidadosa y detallista.

Uno de los aspectos claves para potenciar el interés de los estudiantes por la geometría, y fomentar el logro de competencias puede ser incorporar más ejercicios y trabajos donde puedan vincular los contenidos abstractos con situaciones de 
la vida real. Esto contribuiría a sensibilizar a los estudiantes acerca de la importancia de estos contenidos y a percibir las aplicaciones de la geometría en los diferentes contextos profesionales y de la actividad cotidiana. En este sentido, Fandiño, Díaz y D’Amore (2008) proponen elaborar estrategias didácticas con las cuales los estudiantes se sientan atraídos no solo a examinar cadenas de contenidos, sino a participar en la construcción de sus propias competencias, a partir de conceptos elegidos de manera tal que constituyan un interés por sí mismos y a desarrollos que involucren y amalgamen otros contenidos considerados claves en el desarrollo de la disciplina.

\section{CONCLUSIONES}

Con respecto a las competencias en geometría, de los estudiantes de las escuelas públicas del Municipio Ramiriquí, Departamento de Boyacá, se encontró que tienen un dominio apenas suficiente de las competencias en geometría. Los tres componentes de las competencias (cognitivas, procedimentales y actitudinales) poseen niveles de dominio similares. Además se logró evidenciar una tendencia en cuanto a la dificultad con establecer estrategias para la solución de problemas relativos a la medida del perímetro de figuras planas. También se logró evidenciar que los estudiantes prefieren realizar ejercicios prácticos que sean aplicables en algún contexto específico antes que realizar tareas netamente teóricas.

\section{REFERENCIAS}

Buró (2002). Colombia: Datos mundiales de educación 2010/11.
Proquest.
Recuperado de https://ebookcentral.proquest.com

Castellanos, M. (2001). Tablas y gráficos estadísticos en la prueba Saber de Colombia (Tesis de maestría).Universidad de Granada

D’Amore, B. (2005). Bases filosóficas, pedagógicas, epistemológicas y conceptuales de la didáctica de la matemática. Barcelona: Reverté

Fabbricatore, O. (2009). Currículo de educación básica. Recuperado de: https://ebookcentral.proquest.com

Fandiño, M. Díaz, J. y D’Amore, B. (2008). Competencias y matemáticas. Bogotá: Magisterio

Godino, J y Ruiz, F. (2002). Matemáticas y su didáctica para maestros. Granada, España: Universidad de Granada. Recuperado de https://www.ugr.es/ jgodino/edumatmaestros/manual/4_Geometria.pdf

Gómez y Perry (1996). La problemática de las matemáticas escolares. Un reto para directivos y profesores. Grupo Editorial Iberoamérica. Bogotá Colombia

León y Barcia (2016). Didáctica de la geometría para la escuela primaria. Recuperado de https://ebookcentral .proquest.com

León, G. J. L. (2008). Estrategia didáctica para el desarrollo de habilidades geométricas en el primer ciclo de la educación primaria. Recuperado de https://ebookcentral.proquest.com

Lori, M., Fandiño Pinilla, M. I., y D'Amore, B. (2013). La semiótica en la didáctica de la matemática. Bogotá: Magisterio

Marchesi, A., Tedesco, C. y Coll, C. (2015). Calidad, equidad y reformas en la enseñanza. Madrid, España

MEN. (2004). El desarrollo de la educación en el siglo XXI informe nacional de Colombia. Ministerio de educación nacional. Informe elaborado con el contenido propuesto por la UNESCO 
MEN. (2006). Estándares básicos de Tobón, S. (2006) Formación basada en competencias. Bogotá: Ministerio de Educación Nacional

SEDBOYACA (2018). Educación para la competencias. Pensamiento complejo, diseño curricular y didáctica. Ecoe Ediciones

creatividad y la vida. Histórico de pruebas SABER 